\title{
BMJ Open Comparative survival and role of STS score in aortic paravalvular leak after SAVR or TAVR: a retrospective study from the USA
}

\author{
Ratnasari Padang, ${ }^{1}$ Mahmoud Ali, ${ }^{1}$ Kevin L Greason, ${ }^{2}$ Christopher G Scott, ${ }^{3}$ \\ Manasawee Indrabhinduwat, ${ }^{1}$ Charanjit S Rihal, ${ }^{1}$ Mackram F Eleid, ${ }^{1}$ \\ Vuyisile T Nkomo, ${ }^{1}$ Patricia A Pellikka, ${ }^{1}$ Sorin V Pislaru ${ }^{1}$
}

To cite: Padang R, Ali M, Greason KL, et al. Comparative survival and role of STS score in aortic paravalvular leak after SAVR or TAVR: a retrospective study from the USA. BMJ Open 2018;8:e022437. doi:10.1136/ bmjopen-2018-022437

- Prepublication history for this paper is available online. To view these files, please visit the journal online (http://dx.doi org/10.1136/bmjopen-2018022437).

Received 17 February 2018 Revised 25 August 2018 Accepted 7 November 2018

Check for updates

(c) Author(s) (or their employer(s)) 2018. Re-use permitted under CC BY-NC. No commercial re-use. See rights and permissions. Published by BMJ.

${ }^{1}$ Department of Cardiovascular Diseases, Mayo Clinic,

Rochester, Minnesota, USA

${ }^{2}$ Department of Cardiovascular Surgery, Mayo Clinic, Rochester, Minnesota, USA

${ }^{3}$ Division of Biomedical Statistics and Informatics, Mayo Clinic, Rochester, Minnesota, USA

Correspondence to

Dr Sorin V Pislaru;

pislaru.sorin@mayo.edu

\section{ABSTRACT}

Objective The presence of aortic paravalvular leak (PVL) is associated with lower survival, but a direct comparison of its impact after transcatheter aortic valve replacement (TAVR) versus surgical aortic valve replacement (SAVR)

has not been performed. This study sought to determine the differential influence of PVL on survival following TAVR versus SAVR and in patients with varying levels of risk as defined by the Society of Thoracic Surgeons (STS) risk score.

Methods Patients with and without postprocedural PVL were identified from 2290 patients undergoing TAVR or SAVR at Mayo Clinic between 2008 and 2014. The primary endpoint was overall survival.

Results There were 588 patients with PVL (374 TAVR, 214 SAVR): age $78 \pm 11$ years, $63 \%$ male and mean follow-up of $3 \pm 2$ years. PVL was trivial/mild in $442(75 \%)$ patients. In propensity-matched analyses ( $\mathrm{n}=86 \mathrm{per}$ group), the overall survival at 1 and 4 years was $93 \%$ and $56 \%$ vs $89 \%$ and $61 \%$ in patients with PVL after TAVR versus SAVR, respectively $(\mathrm{p}=0.43)$. The presence or degree of PVL severity had no influence on survival of patients with high STS score $(\geq 8 \%)$, while the presence of greater than mild PVL predicted worse survival in those with STS score $<8 \%$. During the first year after PVL diagnosis, while either improvement or stable PVL grade was seen in the majority of patients, worsening of PVL grade was more common in the TAVR group (19\%) versus the SAVR group (4\%) $(p<0.0001)$.

Conclusions At mid-term follow-up, the presence of PVL was associated with equally unfavourable outcomes following SAVR or TAVR. In patients with high STS risk score, the presence of PVL was not independently associated with increased mortality.

\section{INTRODUCTION}

Symptomatic severe aortic stenosis portends poor outcome, and aortic valve replacement (AVR), either by surgery (SAVR) or transcatheter (TAVR) approach, is recommended. ${ }^{1}$ Paravalvular leak (PVL) is an important complication of AVR which may lead to haemolysis and/or heart failure. ${ }^{2}$ The
Strengths and limitations of this study

- This study was the first to compare the influence of aortic paravalvular leak (PVL) on survival after transcathether (TAVR) versus surgical aortic valve replacement (SAVR), and was the first to determine the relative influence of aortic PVL on survival in patients with varying level of operative risk as determined by the Society of Thoracic Surgeons score.

- Propensity score-matched analysis was used to rigorously adjust for baseline clinical differences between the SAVR and TAVR groups in order to minimise potential confounders.

- The retrospective single-centre study design may have resulted in selection bias.

- Although PVL severity in all cases was graded using the same composite echocardiographic criteria as recommended by the American Society of Echocardiography and the Valve Academic Research Consortium, echocardiography data were not evaluated by a centralised core laboratory; this may have caused some variability in the grading of PVL severity.

incidence of PVL is higher after TAVR (up to $70 \%)$ than SAVR $(2 \%-17 \%) .{ }^{3-5}$ While most PVLs are haemodynamically insignificant, clinically silent and are traditionally thought to have a relatively benign course, ${ }^{6}$ contemporary literature suggests that even mild PVL is associated with worse survival following either TAVR or SAVR. ${ }^{7-9}$ Nevertheless, a direct comparison of the influence of PVL on survival after TAVR versus SAVR has not been reported.

The Society of Thoracic Surgeons (STS) risk score is an algorithm that calculates the impact of patient clinical risk factors on their risk of operative mortality and is widely used to determine TAVR candidacy. Prior studies have shown STS score to be a good predictor for long-term prognosis after successful SAVR 
and TAVR. ${ }^{10}{ }^{11}$ However, the prognostic impact of aortic PVL in patients with varying levels of risk as determined by the STS score has not been studied.

We hypothesised that PVL has a similar influence on survival of patients following either TAVR or SAVR. With the ever-expanding indication for TAVR as an alternative therapy to SAVR for patients with symptomatic severe aortic stenosis, confirmation of this hypothesis would be important as it is likely to influence clinical decision-making process. This study compared the influence of PVL on clinical outcome in patients who develop PVL following TAVR versus SAVR and investigated the predictive role of aortic PVL on survival in patients with varying levels of STS risk.

\section{MATERIALS AND METHODS}

\section{Study population}

All patients aged $>18$ years undergoing TAVR or SAVR \pm coronaryartery bypass grafting (CABG) at Mayo Clinic, Rochester, between November 2008 and October 2014, were retrospectively identified from the cardiac surgery database. Patients with PVL of any degree (trivial to severe) seen on echocardiography at any time point following SAVR/TAVR were identified as the PVL cohort; otherwise they were categorised as the 'no PVL' cohort. A minimum of 1-year clinical follow-up was required for study inclusion unless death occurred earlier. To identify the isolated effect of aortic PVL, patients with concomitant greater than mild aortic prosthetic regurgitation and those with SAVR who received composite aortic root replacement, valve homograft/autograft, concomitant valve surgery at other position(s) and/or aortic surgery were excluded. Patients who developed aortic PVL in the context of preoperative endocarditis were also excluded $(\mathrm{n}=5)$.

Baseline clinical, echocardiographic and procedural/ surgical data were abstracted from electronic medical records. Preprocedural STS scores were calculated using the freely available online calculator (http://riskcalc.sts. $\mathrm{org} /$ stswebriskcalc/\#/). High surgical risk was defined as STS score $\geq 8 \%$ and low/intermediate risk as STS score $<8 \%{ }^{12}$

\section{Echocardiography and PVL grading}

All patients underwent routine intraprocedural transoesophageal echocardiogram and a predismissal transthoracic echocardiogram (TTE). Follow-up TTEs were performed at the discretion of the patient's primary cardiologist, based on clinical indications and as per guideline recommendation. ${ }^{1}$ Aortic PVL was identified as high-velocity jet(s) on colour Doppler imaging originating from a space between the prosthesis sewing ring (post-SAVR) or valve stent (post-TAVR) and the native valve annulus. PVL severity was graded using composite echocardiographic criteria recommended by the American Society of Echocardiography and the Valve Academic Research Consortium, ${ }^{6}{ }^{13}$ which took into account both quantitative and semiquantitative parameters that included left ventricular size, circumferential extent of the regurgitant jet on aortic valve short-axis view, regurgitant volume by either proximal isovelocity surface area method or continuity equation, and assessment of diastolic Doppler flow reversal in descending thoracic aorta. Based on the overall parameters, PVL was graded as trivial, mild, mild-moderate, moderate, moderate-severe or severe.

All available echocardiography reports for each patient were reviewed for the presence of PVL, and when present its severity was recorded based solely on these reports. To understand the natural history of PVL progression in SAVR and TAVR, PVL severity was analysed at the time of PVL diagnosis and at 1-year follow-up after diagnosis. The maximum degree of PVL grading at any echocardiographic time point was used to categorise patients for comparative outcome analysis.

\section{Clinical outcomes}

The primary endpoint was all-cause mortality, determined from the electronic medical record, autopsy reports and/ or Social Security Death Index. The secondary endpoints were postoperative/postprocedural endocarditis, aortic valve reintervention (by redo AVR, valve-in-valve procedure or percutaneous PVL closure), PVL-related haemolysis and rehospitalisation from heart failure. For outcome analyses, patients with PVL were categorised as those with trivial/mild PVL and those with greater than mild PVL; patients without PVL were used as a comparative group.

\section{Patient and public involvement}

Patients and the public were not involved in the development of this study.

\section{Statistical analysis}

Continuous variables were presented as mean \pm SD for normally distributed data, or median (IQR) for skewed data, and compared using two-sided unpaired Student's t-test or Kruskal-Wallis rank-sum test for multiple group comparison; logarithmic transformations were used as appropriate. Categorical variables were presented as counts and percentages, and compared using the $\mathrm{X}^{2}$ or Fisher's exact test as appropriate. Survival/outcome analyses were performed using the Kaplan-Meier method and summarised with corresponding Kaplan-Meier survival estimates, and groups were compared using the log-rank test. When comparing patients with PVL with those without, time 0 represented the time of SAVR or TAVR. When looking at outcomes after diagnosis of PVL, time 0 represented the time of initial PVL diagnosis following SAVR or TAVR. Patients who did not reach the study endpoints or were lost to follow-up were censored at the time of their last clinical follow-up. Cox proportional hazards regression was used to determine the association between variables and mortality after adjusting for other covariates and expressed as HR and 95\% CI.

Meanwhile, to adjust for potential confounders during comparative survival analysis among those with aortic PVL, 
propensity score matching was used to select two homogeneous groups of patients with PVL following TAVR and SAVR, in whom isolated severe aortic stenosis was the preprocedural indication. Subjects were scored based on the propensity of having TAVR or SAVR given the year of PVL diagnosis (to allow comparable follow-up duration between the matched cohort during which PVL could manifest its clinical impact) and the following 15 baseline characteristics: age, sex, PVL severity, STS score, New York Heart Association (NYHA) functional class, history of triple vessels coronary artery disease, prior CABG, stroke, peripheral vascular disease, chronic obstructive pulmonary disease (COPD), mediastinal irradiation, creatinine level, chronic liver disease, pulmonary hypertension and atrial fibrillation. A greedy matching algorithm was then used to match subjects based on their propensity scores, and then analyses were performed within this subset of matched subjects. Standardised mean differences (difference between groups divided by SD) are presented to show balance between groups. Due to the large degree of observed difference in these important factors between groups, not all subjects were able to be matched. A secondary analysis was undertaken using inverse probability weights (IPW). Briefly, this method uses all subjects and weights each subject based on the inverse of the propensity score, so some subjects will have very small or very large weights. The association between SAVR versus TAVR and mortality were estimated using Cox regression for both propensity-matched groups and using IPW.

To study the association of PVL on survival in patients with varying levels of STS risk, the outcome of patients with aortic PVL that developed within a short time frame following their AVR (ie, $\leq 3$ months) was compared with patients without aortic PVL during the same postprocedural time period; the latter group included those who never developed PVL (ie, no PVL group, $\mathrm{n}=1561,1525$ SAVR and 36 TAVR) and those in whom PVL was detected late ( $>3$ months post-AVR, $\mathrm{n}=61,54$ SAVR and 7 TAVR). This was performed to avoid survival bias created by those who develop PVL late following their AVR. Cox regression was used to examine the association of PVL that developed within 3 months with outcomes beyond 3 months, and an interaction between PVL and STS score was examined. As a secondary method to evaluate the association of PVL with outcome, PVL was used as a time-dependent covariate, and the risk of mortality with PVL was estimated using proportional hazards regression.

All statistical analyses were performed using the JMP V.10.0 and SAS V.9.4 software. Two-sided $\mathrm{p}$ value $<0.05$ was considered statistically significant.

\section{RESULTS}

During the study period, 2290 patients underwent either $\mathrm{SAVR} \pm \mathrm{CABG}(\mathrm{n}=1825)$ or TAVR $(\mathrm{n}=465)$. Any degree of PVL was detected in $300(16 \%)$ and $429(92 \%)$ patients following SAVR and TAVR, respectively. Greater than mild PVL was present in $20(1 \%)$ patients post-SAVR and

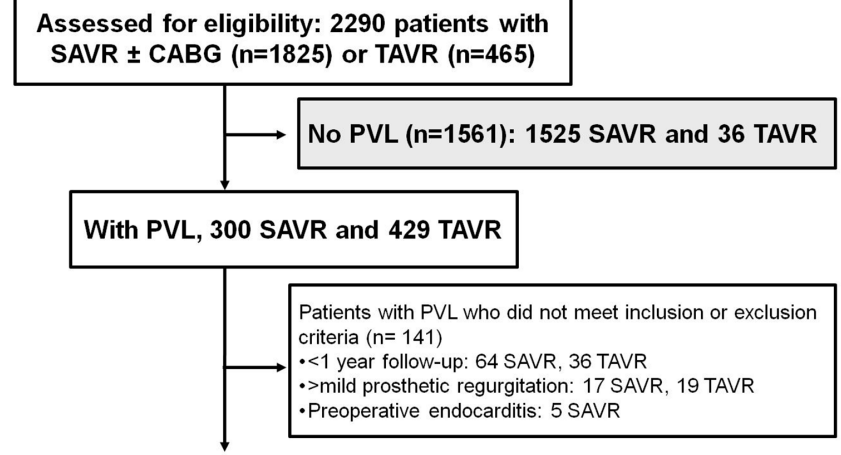

PVL cohort ( $n=588): 214$ SAVR and 374 TAVR

Figure 1 Patient selection criteria. CABG, coronary artery bypass grafting; PVL, paravalvular leak; SAVR, surgical aortic valve replacement; TAVR, transcatheter aortic valve replacement.

in $127(27 \%)$ post-TAVR $(\mathrm{p}<0.0001)$. Of those with aortic PVL, 588 patients (214 SAVR and 374 TAVR) met the inclusion/exclusion criteria and became the PVL study cohort (figure 1).

\section{Characteristics of patients with aortic PVL}

The mean age at time of AVR for the PVL cohort $(n=588)$ was $78 \pm 11$ years; $372(63 \%)$ were male. Compared with the SAVR group (table 1), the TAVR group was older, had a greater proportion of female sex and a higher median STS score. Further, the TAVR cohort had a greater proportion of patients with NYHA class III/IV symptoms, hypertension, coronary artery disease, stroke and COPD. In the SAVR group, bioprosthesis was used in $161(75 \%)$ patients, $<26 \mathrm{~mm}$ prosthesis was used in $93 \%$ of cases, and the maximal PVL severity was trivial/mild in 195 (91\%) patients. In the TAVR group, balloon-expandable valves were used in 347 (93\%) patients, $\geq 26 \mathrm{~mm}$ prosthesis was implanted in $68 \%$ of cases, and the maximal PVL severity was trivial/mild in 247 (66\%) patients.

\section{Time course of PVL detection and its progression}

In the majority of patients with aortic PVL, PVL was identified at the time of implantation, on predischarge TTE or at 3 months postprocedural follow-up (160 [75\%] SAVR and 367 [98\%] TAVR). In the remainder, PVL was detected at follow-up TTE $\sim 1.6$ years postprocedure (range $0.3-6.1$ years); six cases (11\%; all post-SAVR) occurred in the context of endocarditis.

The proportion of patients with PVL in the two groups, categorised based on their severity at time of PVL diagnosis and 1-year follow-up, is summarised in figure 2A. At both time points, greater than mild PVL was more common in the TAVR group. PVL progression in individual patients at 1-year follow-up ( $\mathrm{n}=425 ; 142$ SAVR, 283 TAVR) is summarised in figure 2B. This excluded patients with no echocardiography follow-up at 1 year after PVL diagnosis $(\mathrm{n}=79$; 56 SAVR [26\%], 23 TAVR [6\%]) and those who either died or had aortic valve reintervention within 
Table 1 Baseline characteristics of the aortic PVL cohort $(n=588)$

\begin{tabular}{|c|c|c|c|}
\hline Characteristics & SAVR $(n=214)$ & TAVR $(n=374)$ & $P$ values \\
\hline Age, years & $72 \pm 13$ & $81 \pm 8$ & $<0.0001$ \\
\hline Male sex & $150(70)$ & $222(59)$ & 0.009 \\
\hline Median STS score (IQR), \% & $3.0(1.8-5.0)$ & $7.9(5.5-10.9)$ & $<0.0001$ \\
\hline NYHA class III/IV & $153(72)$ & $334(89)$ & $<0.0001$ \\
\hline Hypertension & $158(74)$ & $336(90)$ & $<0.0001$ \\
\hline Coronary artery disease & $121(57)$ & $267(71)$ & 0.0003 \\
\hline Previous CABG & $94(44)$ & $158(42)$ & 0.69 \\
\hline Cerebrovascular accident & $16(7)$ & $92(25)$ & $<0.0001$ \\
\hline Mediastinal radiation & $9(4)$ & $24(6)$ & 0.25 \\
\hline Chronic liver disease/cirrhosis & $7(3)$ & $13(3)$ & 0.89 \\
\hline \multicolumn{4}{|l|}{ Surgical/Procedural data } \\
\hline \multicolumn{4}{|l|}{ Procedural indications } \\
\hline Aortic stenosis & $186(87)$ & $365(98)$ & \\
\hline Aortic regurgitation & $13(6)$ & $0(0)$ & \\
\hline Bioprosthetic dysfunction & $15(7)$ & $9(2)$ & \\
\hline Valve type $^{*}$ & $\begin{array}{l}\text { Mechanical } 53(25) \\
\text { Bioprosthesis } 161(75)\end{array}$ & $\begin{array}{l}\text { Sapien } \\
\text { Sapien XT } \\
\text { Sapien S3 } \\
\text { CoreValve }\end{array}$ & $\begin{array}{l}211(57) \\
83(22) \\
53(14) \\
27(7)\end{array}$ \\
\hline$\leq$ Mild & $195(91)$ & $247(66)$ & \\
\hline Mild-moderate or moderate & $13(6)$ & $112(30)$ & \\
\hline$>$ Moderate & $6(3)$ & $15(4)$ & $<0.0001$ \\
\hline Prosthetic regurgitation & 67 trivial; 13 mild & 96 trivial; 18 mild & 0.10 \\
\hline LVEF, \% & $59 \pm 11$ & $57 \pm 12$ & 0.03 \\
\hline$\geq$ Moderate RV dysfunction & $19(9)$ & $35(10)$ & 0.79 \\
\hline$\geq$ Moderate MR & $13(6)$ & $81(22)$ & $<0.0001$ \\
\hline$\geq$ Moderate TR & $25(12)$ & $101(28)$ & $<0.0001$ \\
\hline $\mathrm{RVSP} \geq 50 \mathrm{~mm} \mathrm{Hg}$ & $22(11)$ & $105(29)$ & $<0.0001$ \\
\hline LV mass index, $\mathrm{g} / \mathrm{m}^{2}$ & $118 \pm 32$ & $125 \pm 32$ & 0.009 \\
\hline Mean aortic prosthesis gradient, $\mathrm{mm} \mathrm{Hg}$ & $16 \pm 7$ & $12 \pm 5$ & $<0.0001$ \\
\hline Aortic prosthesis EOA, $\mathrm{cm}^{2}$ & $1.98 \pm 0.6$ & $2.24 \pm 0.7$ & $<0.0001$ \\
\hline
\end{tabular}

Unless otherwise specified, values are mean \pm SD or $n(\%)$. Statistically significant $p$-values were typed in bold.

*In patients undoing SAVR, the mechanical valves used were On-X $(n=3 ; 1 \%)$, Carbomedics $(n=29 ; 14 \%)$ and St Jude $(n=21 ; 10 \%)$ valves, while the bioprosthetic valves used were Trifecta $(n=33 ; 15 \%)$, Perceval $(n=5 ; 3 \%)$, Hancock $(n=9 ; 4 \%)$, CE Perimount $(n=52 ; 24 \%)$, Mitroflow $(n=54$; $25 \%)$ and St Jude Epic ( $n=8 ; 4 \%)$ valves.

CABG,coronary artery bypass grafting; EOA, effective orifice area; LV, left ventricle; LVEF, left ventricular ejection fraction; MR, mitral regurgitation; NYHA, New York Heart Association; PVL, paravalvular leak; RV, right ventricle; RVSP, right ventricular systolic pressure; SAVR, surgical aortic valve replacement; STS, Society of Thoracic Surgeons; TAVR, transcatheter aortic valve replacement; TR, tricuspid regurgitation. 

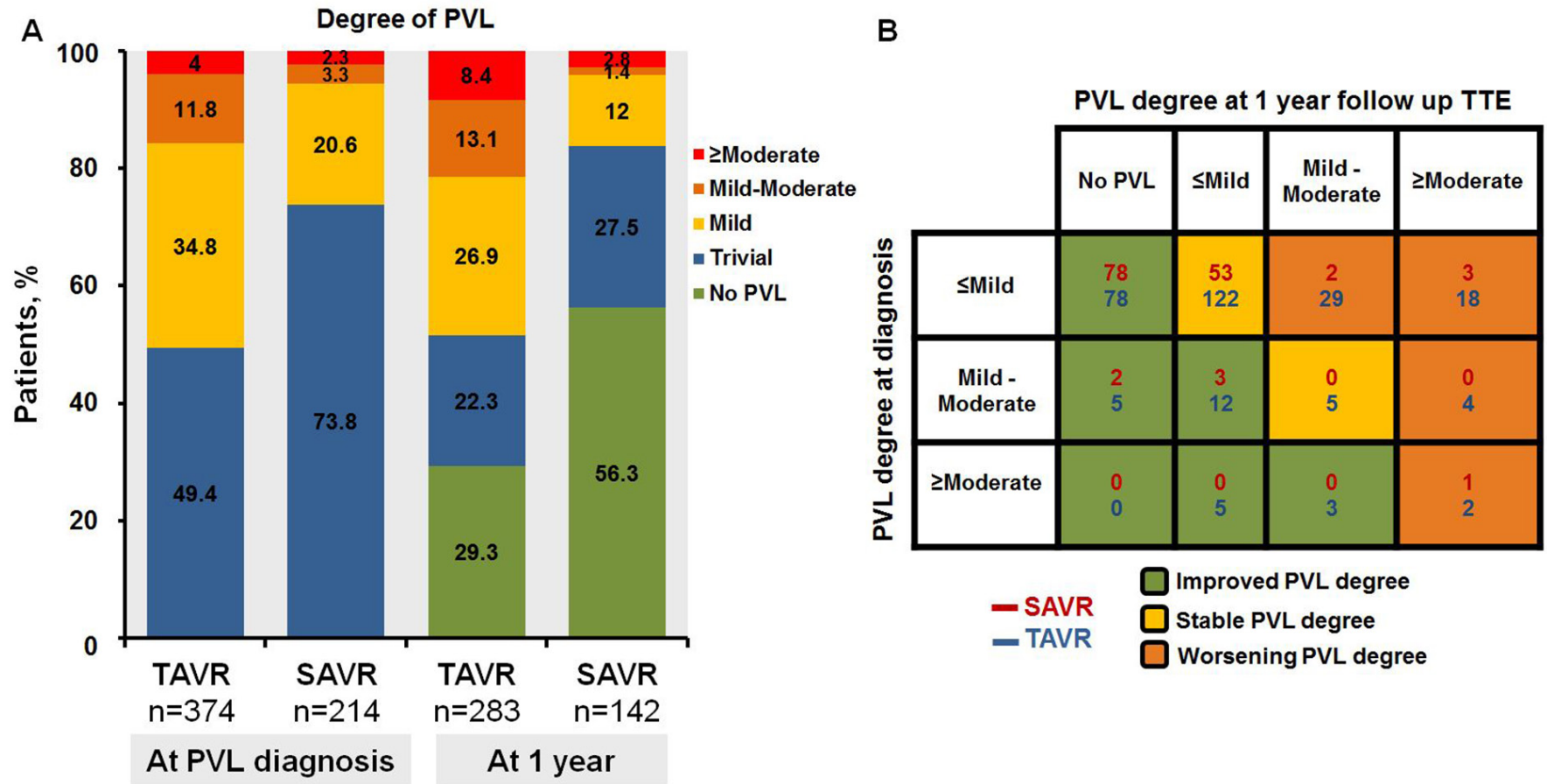

Figure 2 PVL progression in TAVR and SAVR. (A) Patients with PVL at the time of diagnosis and 1-year follow-up in the TAVR and SAVR groups. (B) PVL progression in 425 patients with PVL in the SAVR or TAVR group based on their echocardiographic findings at time of PVL diagnosis and at 1-year follow-up. This excluded patients with no echocardiography follow-up at 1 year postprocedure ( $n=79$; 56 SAVR, 23 TAVR) and those who either died or had aortic valve reintervention within 1 year $(n=84$; 16 SAVR, 68 TAVR). PVL, paravalvular leak; SAVR, surgical aortic valve replacement; TAVR, transcatheter aortic valve replacement; TTE, transthoracic echocardiography.

1 year (n=84; 16 SAVR [8\%], 68 TAVR [18\%]). While PVL remained unchanged or improved in $366(86 \%)$ patients for whom data were available, worsening of PVL by $\geq 1$ grade was more common in TAVR than in SAVR ( $19 \%$ vs $4 \%, \mathrm{p}<0.0001)$.

\section{Outcomes of patients with aortic PVL following SAVR and TAVR}

During a mean follow-up of $3 \pm 2$ years in patients with aortic PVL ( $\mathrm{n}=588$; total follow-up 1551 patient years), there were 214 deaths (47 SAVR, 167 TAVR), of which 50 were cardiac (12 SAVR, 38 TAVR), 52 were non-cardiac (9 SAVR, 43 TAVR), and 112 were death of unascertained cause (26 SAVR, 86 TAVR). There were 15 patients with late postprocedural endocarditis (7 SAVR [3\%], 8 TAVR [2\%], $\mathrm{p}=0.41), 38$ with PVL-related haemolysis (12 SAVR [6\%], 26 TAVR [7\%], $\mathrm{p}=0.52$ ) and 19 who required reintervention for their PVL (8 SAVR [4\%], 11 TAVR [3\%], $\mathrm{p}=0.63$ ).

On initial analysis of the PVL cohort, the presence of PVL seemed to be associated with worse survival after TAVR compared with SAVR. Among these two heterogeneous groups of patients with aortic PVL (table 1), survival was $93 \%, 84 \%$ and $73 \%$ vs $83 \%, 59 \%$ and $30 \%$ at 1,3 and 5 years in SAVR versus TAVR, respectively $(\mathrm{p}<0.0001)$. And while the risk for endocarditis, valve reintervention or PVL-related haemolysis was similar in both groups, the rate of rehospitalisation from heart failure at 5 years was higher in the TAVR than in the SAVR group (20.2\% vs $9.8 \%$, respectively, $\mathrm{p}=0.02$; table 2 ). Notably, the presence of haemolysis per se was not associated with increased risk of death in either group ( $\mathrm{p}>0.5$ for both).

However, no difference in overall survival was seen in the propensity-matched analysis, which included 86 homogeneous pairs of patient with aortic PVL in each group with comparable baseline characteristics (table 3 ). In the propensity-matched cohort, the probability of survival was similar at $89 \%$ and $61 \%$ vs $93 \%$ and $56 \%$ at 1 and 4 years in patients with aortic PVL after SAVR versus TAVR, respectively (log-rank $\mathrm{p}=0.43$, adjusted HR 1.15 , $95 \%$ CI 0.69 to 1.93; figure 3). Additionally, a secondary analysis was performed using IPW so that all subjects with aortic PVL were retained; reassuringly, the adjusted HR for mortality was found to be very similar to the propensity-matched analysis (adjusted HR using IPW analysis 1.16, $95 \%$ CI 0.86 to $1.58, \mathrm{p}=0.33$ ). No significant difference was observed among the propensity-matched cohort for the development of secondary endpoints.

\section{Influence of baseline aortic regurgitation on survival of} patients with aortic PVL

Among patients with mild or greater aortic PVL ( $n=336)$, the presence of baseline moderate or greater aortic regurgitation $(\mathrm{AR})$ was associated with improved survival 
Table 2 Clinical outcomes in patients with PVL following SAVR versus TAVR based on Kaplan-Meier estimates at specific time points

\begin{tabular}{|c|c|c|c|}
\hline & $\begin{array}{l}\text { SAVR } \\
(n=214), \%\end{array}$ & $\begin{array}{l}\text { TAVR } \\
(\mathrm{n}=374), \%\end{array}$ & Log-rank $p$ values \\
\hline \multicolumn{4}{|c|}{ Overall mortality } \\
\hline At 1 year & 6.8 & 17.4 & \multirow[t]{3}{*}{$<0.0001$} \\
\hline At 3 years & 16.4 & 41.3 & \\
\hline At 5 years & 26.7 & 69.7 & \\
\hline \multicolumn{4}{|l|}{ Endocarditis } \\
\hline At 1 year & 2.4 & 0.9 & \multirow[t]{3}{*}{0.69} \\
\hline At 3 years & 3.1 & 3.4 & \\
\hline At 5 years & 5.8 & 3.4 & \\
\hline \multicolumn{4}{|c|}{ PVL-related haemolysis } \\
\hline At 1 year & 2.4 & 3.8 & \multirow[t]{3}{*}{0.19} \\
\hline At 3 years & 6.2 & 7.9 & \\
\hline At 5 years & 7.8 & 14.4 & \\
\hline \multicolumn{4}{|c|}{ Rehospitalisation from heart failure } \\
\hline At 1 year & 4.7 & 9.3 & \multirow[t]{3}{*}{0.02} \\
\hline At 3 years & 9.8 & 14.6 & \\
\hline At 5 years & 9.8 & 20.2 & \\
\hline \multicolumn{4}{|c|}{ Aortic valve reintervention } \\
\hline At 1 year & 1.9 & 1.1 & \multirow[t]{3}{*}{0.93} \\
\hline At 3 years & 3.8 & 3.0 & \\
\hline At 5 years & 4.8 & 9.1 & \\
\hline
\end{tabular}

PVL, paravalvular leak; SAVR, surgical aortic valve replacement; TAVR, transcatheter aortic valve replacement.

compared with those with none or less than moderate AR prior to their AVR (HR 0.57, CI 0.33 to $0.92, \mathrm{p}=0.02$ ).

\section{Association of aortic PVL with survival and role of STS score}

Patients with PVL diagnosed within 3 months following SAVR/TAVR had worse survival compared with the corresponding group without PVL at the same postprocedural time point; those with greater than mild PVL had the worst outcome (figure 4A). Notably, the median STS scores were $2.3 \%, 5.9 \%$ and $7.0 \%$ in the overall cohorts with no PVL, trivial/mild and greater than mild PVL, respectively $(p<0.007)$. Thus, to evaluate the influence of STS scores on the outcome of patients with aortic PVL, patients were divided into those with low/intermediate STS and those with high STS groups, and their outcome compared based on PVL presence and severity (figure 4B).

A notable difference was observed on the influence of PVL among the different STS subgroups. The interaction between PVL severity and STS subgroups was tested with Cox regression and found to be significant (age-adjusted and gender-adjusted $\mathrm{p}=0.009$ ), suggesting a differential effect of PVL depending on STS. PVL severity was not associated with differential survival among patients with high STS score $(\mathrm{n}=321$, median STS $10.9 \%$; log-rank $\mathrm{p}=0.80$ ), who as a group had an overall 5-year survival of $26 \%$. Meanwhile among those with low/intermediate STS score $(\mathrm{n}=1828$, median STS 2.3\%), the presence of greater than mild PVL was associated with worse survival compared with those without PVL (adjusted HR 2.0, CI 1.3 to $2.8, \mathrm{p}=0.001$ ) and those with trivial $/$ mild PVL (adjusted HR 1.5, CI 1.0 to 2.3, $\mathrm{p}=0.05$ ). Survival between those without PVL and those with trivial/mild PVL in the low/intermediate STS group was comparable (age-adjusted/gender-adjusted $\mathrm{p}=0.10$ ).

Notably, aortic PVL of any degree was present in 386 $(21 \%)$ patients in the low/intermediate STS group, and was greater than mild in severity in $92(5 \%)$ patients (16\% SAVR, $84 \%$ TAVR). In contrast, aortic PVL of any degree was present in $202(63 \%)$ patients in the high STS group, which was greater than mild in severity in 54 $(17 \%)$ patients $(7 \%$ SAVR, 93\% TAVR) $(\mathrm{p}<0.0001)$.

\section{DISCUSSION}

The following were the main findings of this study of patients with aortic PVL: (1) PVL portended equally unfavourable influence on survival following SAVR or TAVR. (2) PVL was three times more common among patients with high than those with low/intermediate STS score. (3) PVL was not associated with differential survival in patients with high STS scores $(\geq 8 \%)$. In contrast, the presence of greater than mild PVL was associated with increased mortality at follow-up in those with low/intermediate risk (STS $<8 \%$ ). (4) During the first year after 
Table 3 Baseline characteristics of the propensity-matched cohort with aortic PVL

\begin{tabular}{|c|c|c|c|c|}
\hline Characteristics & $\begin{array}{l}\text { SAVR } \\
(n=86)\end{array}$ & $\begin{array}{l}\text { TAVR } \\
(n=86)\end{array}$ & $\begin{array}{l}\text { Standardised mean } \\
\text { difference }\end{array}$ & $P$ values \\
\hline \multicolumn{5}{|l|}{ Clinical data } \\
\hline Age, years & $77 \pm 11$ & $78 \pm 9$ & 0.14 & 0.37 \\
\hline Male sex & $55(64)$ & $57(66)$ & 0.05 & 0.75 \\
\hline Median STS score (IQR), \% & $5.0(3.0-7.0)$ & $4.9(3.0-8.0)$ & 0.05 & 0.75 \\
\hline Aortic stenosis as procedural indication & $86(100)$ & $86(100)$ & - & - \\
\hline \multicolumn{5}{|l|}{ PVL severity } \\
\hline Trivial or mild PVL & $75(87)$ & $72(84)$ & 0.10 & 0.52 \\
\hline Greater than mild PVL & $11(13)$ & $14(16)$ & & \\
\hline NYHA class III or IV & $72(84)$ & $71(83)$ & 0.03 & 0.84 \\
\hline Atrial fibrillation & $30(35)$ & $24(28)$ & 0.15 & 0.32 \\
\hline Diabetes & $26(30)$ & $38(44)$ & 0.29 & 0.06 \\
\hline Hypertension & $69(80)$ & $79(92)$ & 0.34 & 0.03 \\
\hline Coronary artery disease & $64(74)$ & $63(73)$ & 0.03 & 0.86 \\
\hline Triple vessels coronary artery disease & $32(37)$ & $38(44)$ & 0.14 & 0.35 \\
\hline Previous CABG & $48(56)$ & $46(53)$ & 0.05 & 0.76 \\
\hline Prior cerebrovascular accident & $11(13)$ & $12(14)$ & 0.03 & 0.82 \\
\hline Peripheral vascular disease & $25(29)$ & $29(34)$ & 0.10 & 0.51 \\
\hline Creatinine level, mg/dL & $1.22 \pm 0.75$ & $1.22 \pm 0.70$ & 0.01 & 0.99 \\
\hline History of endocarditis & $0(0)$ & $0(0)$ & - & - \\
\hline Chronic obstructive airway disease & $23(27)$ & $26(30)$ & 0.08 & 0.61 \\
\hline Mediastinal radiation & $5(6)$ & $6(7)$ & 0.05 & 0.76 \\
\hline Chronic liver disease/cirrhosis & $2(2)$ & $5(6)$ & 0.18 & 0.25 \\
\hline Warfarin therapy & $35(41)$ & $29(35)$ & 0.13 & 0.41 \\
\hline Body mass index, $\mathrm{kg} / \mathrm{m}^{2}$ & $29 \pm 6$ & $31 \pm 7$ & 0.26 & 0.09 \\
\hline \multicolumn{5}{|l|}{ Echocardiographic data } \\
\hline LVEF, \% & $57 \pm 13$ & $58 \pm 11$ & 0.07 & 0.64 \\
\hline$\geq$ Moderate RV dysfunction & $8(10)$ & $9(11)$ & 0.05 & 0.74 \\
\hline$\geq$ Moderate MR & $7(8)$ & $8(9)$ & 0.04 & 0.77 \\
\hline $\mathrm{RVSP} \geq 50 \mathrm{~mm} \mathrm{Hg}$ & $13(15)$ & $17(21)$ & 0.14 & 0.36 \\
\hline Mean aortic prosthesis gradient $(\mathrm{mm} \mathrm{Hg})$ & $16 \pm 6$ & $13 \pm 5$ & 0.47 & 0.002 \\
\hline Aortic prosthesis EOA, $\mathrm{cm}^{2}$ & $1.89 \pm 0.56$ & $2.21 \pm 0.67$ & 0.52 & $<0.001$ \\
\hline
\end{tabular}

Unless otherwise specified, values are mean \pm SD or $n(\%)$. Statistically significant $\mathrm{p}$ values and standardised difference $>0.3$ are printed in bold. Abbreviations as per table 1.

PVL diagnosis, while improvement or stable PVL grade was seen in majority of patients, worsening of PVL grade was more common after TAVR than SAVR. (5) The presence of baseline moderate or greater preoperative AR was associated with improved survival among patients who developed mild or greater aortic PVL following their AVR.

PVL is a potentially serious complication following AVR. Multiple studies have independently shown its adverse influence on survival following SAVR and TAVR. ${ }^{5} 7912$ However, whether patients with aortic PVL have differential outcomes when PVL developed following SAVR versus TAVR was not previously known. Data from the Placement of Aortic Transcatheter Valve Trial (PARTNER1) and PARTNER2 trials suggested that mild or greater and moderate or greater PVL post-TAVR for high-risk and intermediate-risk patients with aortic stenosis, respectively, were associated with lowered survival at medium-term follow-up. ${ }^{9}{ }^{12}$ However, no outcome comparison was made to the SAVR cohort with PVL in these studies, likely due to the small number of patients with moderate or greater PVL following SAVR in these trials.

This study suggested that PVL was associated with equally lowered survival following SAVR or TAVR. While not completely unexpected, this finding is important in light of ongoing expansion of TAVR indications to those with less 


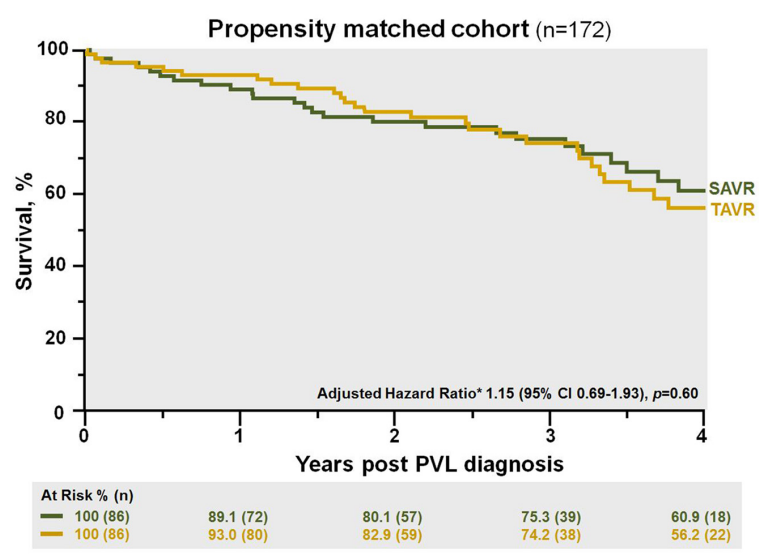

Figure 3 All-cause mortality in patients with PVL stratified by SAVR or TAVR in the propensity-matched cohort. *Adjusted for age, sex, STS score and degree of PVL. PVL, paravalvular leak; SAVR, surgical aortic valve replacement; STS, Society of Thoracic Surgeons; TAVR, transcatheter aortic valve replacement.

than high surgical risk. Since survival of patients with aortic PVL was equally unfavourable following SAVR or TAVR, and as TAVR had been shown to be a good alternative to surgery in both high-risk and intermediate-risk patients, ${ }^{9} 1214$ assessment of patients being considered for AVR should routinely take into account their risk of developing PVL by either SAVR or TAVR. Patients with larger aortic annulus, extensive and asymmetric annular and/or aortic valve calcifications, and bicuspid aortic valve who are at increased risk of developing PVL post-TAVR ${ }^{5}$ may at present be better served with SAVR, particularly in the presence of intermediate or lower surgical risk. Surgical techniques should also be considered at time of SAVR; for example, Silzone cuff (now withdrawn) has been identified as a risk factor for PVL, while the use of pledgets is recommended given their protective effect against PVL. ${ }^{15}$ Ongoing advancement of the current TAVR technologies however will likely overcome some of these anatomical challenges in the future. Meanwhile among those with high surgical risk and favourable valve anatomy, TAVR may be the preferred strategy, particularly given recent encouraging data that suggested an improved 3-year clinical outcomes, including survival, of high-risk patients with aortic stenosis who underwent TAVR with self-expanding valve compared with surgery. $^{14}$

A major finding of this study was that aortic PVL was not an independent predictor of mortality in patients with high STS score $(\geq 8 \%)$. This was not surprising given the robustness of STS score in predicting mortality in patients undergoing either SAVR or TAVR ${ }^{1117}$ and was concordant with prior study that reported STS score to be the strongest predictor of long-term survival following TAVR. ${ }^{11}$ It is likely that patients with higher STS scores succumb to their underlying comorbidities rather than from the PVL, the impact of which may take longer to
A

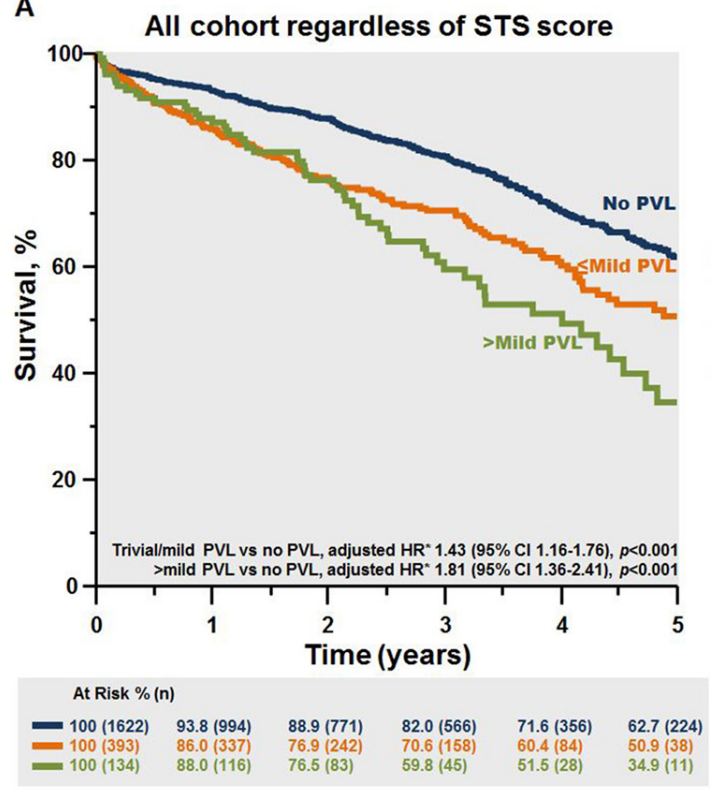

B

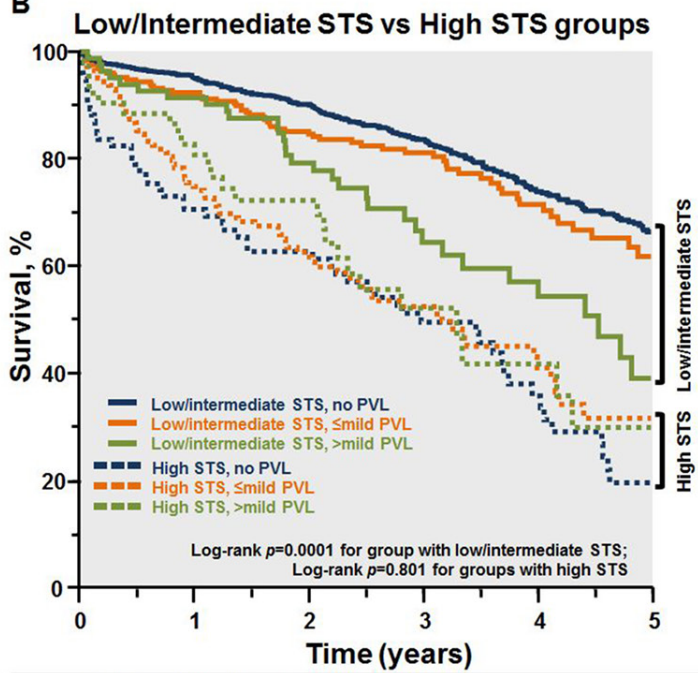

\begin{tabular}{|c|c|c|c|c|c|}
\hline \multicolumn{6}{|c|}{ Lowintermediate STS groups $(<8 \%)$ - At Risk $\%(n)$} \\
\hline 1496) & $\begin{array}{l}95.8(920) \\
92.4(230) \\
91.4(74)\end{array}$ & $\begin{array}{l}90.9(710) \\
84.6(172) \\
79.3(54)\end{array}$ & & $\begin{array}{l}75.0(336) \\
71.5(63) \\
57.1(21)\end{array}$ & $\begin{array}{l}67.1 \text { (216) } \\
61.8(31) \\
39.0(9)\end{array}$ \\
\hline \\
\hline & $\begin{array}{l}74.771 \\
74.811 \\
82.714\end{array}$ & $\begin{array}{l}\mathbf{6 8 . 4}(61) \\
63.4(70) \\
72.2(29)\end{array}$ & $\begin{array}{l}56.4(42) \\
52.1(44) \\
52.1(15)\end{array}$ & $\begin{array}{l}40.2(20) \\
40.9(21) \\
41.7(7)\end{array}$ & $\begin{array}{l}22.9(8) \\
31.5(7) \\
29.8(2)\end{array}$ \\
\hline
\end{tabular}

Figure 4 Influence of PVL on survival based on STS risk score. (A) In the entire AVR cohort (after SAVR or TAVR), with and without aortic PVL. A very similar result was obtained when PVL was used as a time-varying covariate: the time-dependent HR for $\leq$ mild PVL versus no PVL was $1.42(95 \% \mathrm{Cl} 1.16$ to 1.74) and the time-dependent HR for greater than mild PVL versus no PVL groups was 1.83 (95\% Cl 1.39 to 2.41). (B) In the entire AVR cohort after stratification of STS score into those with low/ intermediate versus high STS groups. *Adjusted for age and sex. AVR, aortic valve replacement; PVL, paravalvular leak; SAVR, surgical aortic valve replacement; STS, Society of Thoracic Surgeons; TAVR, transcatheter aortic valve replacement. 
become clinically relevant. On the contrary, the degree of PVL severity did play a role in influencing the outcome of those with low/intermediate STS scores, where the presence of greater than mild PVL portended worse prognosis. This finding was in line with prior reports that suggested the presence of residual mild or greater AR to be an independent predictor for mortality following SAVR $^{7}$ and TAVR. ${ }^{812}$

Another noteworthy finding was that aortic PVL of any degree was present in two-thirds of patients with high STS score, which was three times more common than those with low/intermediate risk. Similarly, greater than mild PVL occurred thrice more common in the highrisk group compared with those with low/intermediate STS score. This suggests that PVL occurrence may be a marker of underlying comorbidities and high STS score. It is plausible that sicker patients (ie, those with higher STS score) may have weaker tissue quality due to chronic procatabolic state and/or impaired synthetic reserve, which augmented the risk of tissue breakdown around suture sites and PVL development postoperatively.

Our study also showed the presence of baseline moderate or greater preoperative AR to be protective on outcome of patients who develop mild or greater PVL following their AVR. A possible explanation for this observation is that the left ventricle (LV) of patients with moderate or greater degree of AR prior to their AVR had undergone adaptive remodelling to compensate for the chronic volume overload. Therefore, when these patients with chronic AR develop greater than mild PVL following their AVR, either by TAVR or SAVR, these patients were able to tolerate the excess volume better compared with those with pure aortic stenosis, who had developed concentric LV hypertrophy due to the chronic increase in afterload preoperatively but who now faced a sudden increase in LV volume due to their PVL.

Reassuringly, improvement or stable aortic PVL grade was seen in the majority (86\%) of study cohort with aortic PVL. This was concordant with findings from the PARTNER1 trial. ${ }^{18}$ Meanwhile, this study observed worsening of PVL grade to be more common following TAVR than SAVR. This finding was supported by data from the PARTNER2 trial, which showed a doubling in the proportion of patient with more than mild PVL post-TAVR at 2years follow-up compared with baseline, while the proportion of these patients was relatively unchanged in the SAVR group. ${ }^{12}$ The mechanisms behind worsening of PVL grade post-TAVR has not been fully elucidated. While this could in part be due to the semiquantitative method used in PVL evaluation, other possible explanations include late endocarditis, confounding by indication (ie, the fact that TAVR patients were generally sicker group of patients with weaker tissue quality), late migration of TAVR valves or late recoil of balloon-expandable TAVR valve stents. ${ }^{19} 20$

Beyond attempting to minimise the occurrence of PVL by various interventions (CT characterisation of aortic valve and annular calcifications, appropriate valve sizing, use of newer generation TAVR valves with sealing mechanisms, and so on), this study suggested that tailoring therapy for PVL should also take into account patients' individual risk. Indeed, percutaneous interventions for PVL in patients with high STS score should only be considered in the presence of symptoms, as the presence of PVL per se was not associated with impaired survival in this high-risk cohort. Prevention, early detection and aggressive treatment of endocarditis are also important to avoid premature structural valve degeneration or late PVL development.

\section{Study limitations}

This retrospective single-centre study may have resulted in selection bias. The study period occurred when TAVR was performed in high-risk population, when early generation balloon-expandable valves were used in majority of cases and prior to the routine use of CT to assist with preprocedural sizing, which may contribute to the higher proportion of greater than mild PVL seen post-TAVR and the higher mortality rate seen in this group. Nevertheless, the 5-year mortality of our TAVR cohort with PVL was similar to that of PARTNER1 trial. ${ }^{9}$ To overcome this limitation, we performed propensity-matched analyses to minimise the difference in baseline characteristics between the two groups to allow meaningful comparisons. Although the matched number of patients was relatively small, the comparative groups were fairly homogeneous, and our analysis yielded a number of important findings in this previously understudied area. Further, it is unlikely that a randomised controlled trial would ever be performed to address this question for obvious ethical reasons.

Specific causes of mortality were only available in $\sim 50 \%$ of subjects and only all-cause mortality could be assessed. Additionally, PVL severity was not a static variable; a proportion of patients experienced either regression or progression of their PVL severity, which may impact their outcome over time. It was however beyond the scope of this study to characterise the impact of PVL progression/regression on clinical outcome. PVL severity was recorded solely based on available echocardiography reports. Further, echocardiography data were not evaluated by a centralised core laboratory, which may cause some variability in the grading of PVL severity. However, in all cases, the same composite criteria was used for PVL grading; thus, any bias introduced in PVL grading of SAVR patients will be balanced by similar bias during grading of TAVR patients.

\section{CONCLUSIONS}

This retrospective analysis demonstrated that the presence of PVL was associated with equally unfavourable outcome following SAVR or TAVR. And in contrast to patients with low/intermediate risk in whom the presence of greater than mild PVL was associated with reduced survival at follow-up, among patients with high surgical risk (STS score $\geq 8 \%$ ) aortic PVL was not independently associated 
with increased mortality. These findings emphasise the importance of individualised patient assessment, from their pre-AVR evaluation, where strategy that is associated with the lowest risk of PVL development should be favoured, to their postprocedural follow-up, where the individual's risk should be routinely considered when tailoring therapy for PVL closure.

Contributors RP and MA were responsible for the design, data collection, analysis, interpretation of data, and drafting and revising the manuscript. CS was responsible for data and statistical analysis. KG, CSR, ME, VN and PAP were responsible for critical revision of the manuscript for important intellectual content, and MI for critical data collection and analysis during manuscript revision. SP was responsible for the design, interpretation of data, critical revision of the manuscript and final approval of the manuscript.

Funding The authors have not declared a specific grant for this research from any funding agency in the public, commercial or not-for-profit sectors.

Competing interests None declared.

Patient consent Not required.

Ethics approval This study was approved by the Mayo Clinic Institutional Review Board Committee.

Provenance and peer review Not commissioned; externally peer reviewed.

Data sharing statement № additional data available.

Open access This is an open access article distributed in accordance with the Creative Commons Attribution Non Commercial (CC BY-NC 4.0) license, which permits others to distribute, remix, adapt, build upon this work non-commercially, and license their derivative works on different terms, provided the original work is properly cited, appropriate credit is given, any changes made indicated, and the use is non-commercial. See: http://creativecommons.org/licenses/by-nc/4.0/.

\section{REFERENCES}

1. Nishimura RA, Otto CM, Bonow RO, et al. 2014 AHA/ACC guideline for the management of patients with valvular heart disease: a report of the American College of Cardiology/American Heart Association Task Force on Practice Guidelines. J Am Coll Cardiol 2014;63:e57-185.

2. Kliger $\mathrm{C}$, Eiros R, Isasti $\mathrm{G}$, et al. Review of surgical prosthetic paravalvular leaks: diagnosis and catheter-based closure. Eur Heart $J$ 2013;34:638-49.

3. Duncan BF, McCarthy PM, Kruse J, et al. Paravalvular regurgitation after conventional aortic and mitral valve replacement: $A$ benchmark for alternative approaches. J Thorac Cardiovasc Surg 2015;150:860-8.

4. Saia F, Martinez C, Gafoor S, et al. Long-term outcomes of percutaneous paravalvular regurgitation closure after transcatheter aortic valve replacement: a multicenter experience. JACC CardiovasC Interv 2015;8:681-8.

5. Sinning JM, Vasa-Nicotera M, Chin D, et al. Evaluation and management of paravalvular aortic regurgitation after transcatheter aortic valve replacement. J Am Coll Cardiol 2013;62:11-20.

6. Zoghbi WA, Chambers JB, Dumesnil JG, et al. Recommendations for evaluation of prosthetic valves with echocardiography and doppler ultrasound: a report From the American Society of Echocardiography's Guidelines and Standards Committee and the Task Force on Prosthetic Valves, developed in conjunction with the American College of Cardiology Cardiovascular Imaging Committee, Cardiac Imaging Committee of the American Heart Association, the European Association of Echocardiography, a registered branch of the European Society of Cardiology, the Japanese Society of Echocardiography and the Canadian Society of Echocardiography, endorsed by the American College of Cardiology Foundation, American Heart Association, European Association of Echocardiography, a registered branch of the European Society of Cardiology, the Japanese Society of Echocardiography, and Canadian Society of Echocardiography. J Am Soc Echocardiogr 2009;22:975-1014. Quiz 82-4.

7. Sponga S, Perron J, Dagenais F, et al. Impact of residual regurgitation after aortic valve replacement. Eur J Cardiothorac Surg 2012;42:486-92.

8. Jones BM, Tuzcu EM, Krishnaswamy A, et al. Prognostic significance of mild aortic regurgitation in predicting mortality after transcatheter aortic valve replacement. J Thorac Cardiovasc Surg 2016;152:783-90.

9. Mack MJ, Leon MB, Smith CR, et al. 5-year outcomes of transcatheter aortic valve replacement or surgical aortic valve replacement for high surgical risk patients with aortic stenosis (PARTNER 1): a randomised controlled trial. Lancet 2015;385:2477-84.

10. Brennan JM, Edwards FH, Zhao Y, et al. Long-term survival after aortic valve replacement among high-risk elderly patients in the United States: insights from the Society of Thoracic Surgeons Adult Cardiac Surgery Database, 1991 to 2007. Circulation 2012;126:1621-9.

11. Hemmann K, Sirotina M, De Rosa S, et al. The STS score is the strongest predictor of long-term survival following transcatheter aortic valve implantation, whereas access route (transapical versus transfemoral) has no predictive value beyond the periprocedural phase. Interact Cardiovasc Thorac Surg 2013;17:359-64.

12. Leon MB, Smith CR, Mack MJ, et al. Transcatheter or surgical aortic-valve replacement in intermediate-risk patients. $N$ Engl $\mathrm{J}$ Med 2016;374:1609-20.

13. Kappetein AP, Head SJ, Généreux $P$, et al. Updated standardized endpoint definitions for transcatheter aortic valve implantation: the valve academic research consortium-2 consensus document. J Am Coll Cardiol 2012;60:1438-54.

14. Deeb GM, Reardon MJ, Chetcuti S, et al. 3-Year Outcomes in highrisk patients who underwent surgical or transcatheter aortic valve replacement. J Am Coll Cardiol 2016;67:2565-74.

15. Englberger L, Schaff HV, Jamieson WR, et al. Importance of implant technique on risk of major paravalvular leak (PVL) after St. Jude mechanical heart valve replacement: a report from the Artificial Valve Endocarditis Reduction Trial (AVERT). Eur J Cardiothorac Surg 2005;28:838-43.

16. Dewey TM, Brown D, Ryan WH, et al. Reliability of risk algorithms in predicting early and late operative outcomes in high-risk patients undergoing aortic valve replacement. J Thorac Cardiovasc Surg 2008;135:180-7.

17. Wendt D, Osswald BR, Kayser K, et al. Society of Thoracic Surgeons score is superior to the EuroSCORE determining mortality in high risk patients undergoing isolated aortic valve replacement. Ann Thorac Surg 2009;88:468-75. Discussion 74-5.

18. Kodali SK, Williams MR, Smith CR, et al. Two-year outcomes after transcatheter or surgical aortic-valve replacement. $N$ Engl J Med 2012;366:1686-95

19. Généreux P, Head SJ, Hahn R, et al. Paravalvular leak after transcatheter aortic valve replacement: the new Achilles' heel? A comprehensive review of the literature. J Am Coll Cardiol 2013;61:1125-36.

20. Karimi A, Pourafshar N, Park KE, et al. Late paravalvular aortic regurgitation: migration of the valve or late recoil? Cardiol Ther 2017;6:133-8. 